\title{
X-linked intellectual disability, Nascimento type
}

INSERM

\section{Source}

INSERM. (1999). Orphanet: an online rare disease and orphan drug data base. $\underline{x \text {-linked }}$ intellectual disability, Nascimento type. ORPHA:163956

X-linked intellectual disability, Nascimento type is a rare X-linked intellectual disability syndrome characterized by intellectual disability (with severe speech impairment), a myxedematous appearance, dysmorphic facial features (including large head, synophrys, prominent supraorbital ridges, almond-shaped and deep-set eyes, large ears, wide mouth with everted lower lip and downturned lip corners), low posterior hairline, short, broad neck, marked general hirsutism and abnormal hair whorls, skin changes (e.g. dry skin or hypopigmented spots), widely spaced nipples, obesity, micropenis, onychodystrophy and seizures. 